\title{
Apolipoprotein L1 Testing in African Americans: Involving the Community in Policy Discussions
}

\author{
Bessie A. Young ${ }^{a} \quad$ Erika Blacksher ${ }^{b}$ Kerri L. Cavanaugh ${ }^{c}$ \\ Barry I. Freedman ${ }^{d}$ Stephanie M. Fullerton ${ }^{b}$ Jeffrey B. Kopp ${ }^{\mathrm{e}}$ \\ Ebele M. Umeukeje ${ }^{c}$ Kathleen M. West ${ }^{b}$ James G. Wilson ${ }^{f}$ \\ Wylie Burke ${ }^{b}$ APOL1 Stakeholders Project
}

a Department of Medicine, VA Puget Sound Health Care System, Division of Nephrology, and Kidney Research Institute, University of Washington, Seattle, WA, USA; ${ }^{b}$ Department of Bioethics and Humanities, University of Washington, Seattle, WA, USA; ${ }^{c}$ Division of Nephrology, Department of Medicine, Vanderbilt University Medical Center, Nashville, TN, USA; d Department of Internal Medicine, Section on Nephrology, Wake Forest School

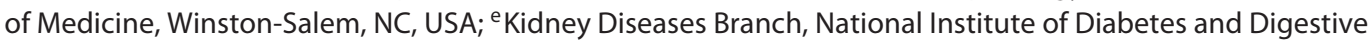
and Kidney Diseases, Bethesda, MD, USA; ${ }^{\mathrm{f}}$ Department of Physiology and Biophysics, University of Mississippi, Jackson, MS, USA

\section{Keywords}

Apolipoprotein L 1 testing · Stakeholder engagement .

End-stage kidney disease

\begin{abstract}
Background: Apolipoprotein A1 (APOL1) gene variants occurring in people of West African descent contribute to the greater burden of kidney disease among African Americans. These variants are associated with increased risk of nondiabetic nephropathy, more rapid progression of chronic kidney disease, and shorter survival of donor kidneys after transplantation. However, only a minority of people with APOL 1-associated risk develops kidney disease and specific clinical measures to address APOL1-associated risk are lacking. Given these uncertainties, we sought to engage members of the African American public in discussions with other stakeholders about the appropriate use of APOL 1 testing. Methods: Formative interviews with community members,
\end{abstract}

(๑) 2019 Published by S. Karger AG, Basel

E-Mail karger@karger.com www.karger.com/ajn researchers, and clinicians in Seattle WA, Nashville TN, and Jackson MS, provided baseline information about views toward APOL 1 testing and informed the design of 3 community-based deliberations among African Americans. A national meeting held in March 2018 included 13 community members, 7 scientific advisors and 26 additional researchers, clinicians, bioethicists, patient advocates, and representatives from professional organizations and federal funding agencies. Using small break-out and plenary discussion, the group agreed on recommendations based on current knowledge about APOL 1-associated risk. Results: Meeting outcomes included recommendations to develop educational materials about APOL 1 for community members and clinicians; to offer $A P O L 1$ research results to participants; and on the use of $A P O L 1$ testing in kidney transplant programs. The group rec-

This is a work of the U.S. Government and is not subject to copyright protection in the United States. Foreign copyrights may apply. 
ommended against the routine offer of $A P O L 1$ testing in clinical care. Areas of disagreement included whether kidney transplant programs should require $A P O L 1$ testing of prospective living donors or bar individuals with $A P O L 1$ risk from donating kidneys and whether testing should be available on request in routine clinical care. Conclusion: We recommend continued discussion among stakeholders and concerted efforts to ensure active and informed participation of members of the affected community to guide research on APOL1 and kidney disease.

(c) 2019 Published by S. Karger AG, Basel

\section{Introduction}

Rapid progress in genomic research is leading to the identification of many gene variants associated with important health outcomes. Often the initial data are provisional and there may be considerable uncertainty about the clinical utility of genetic testing. Clinicians and policy makers face difficult questions in this context: When is information sufficient to introduce a new genetic test into clinical practice? What values and objectives should drive test use; who decides; and what informational resources are required for responsible test use?

The association of the apolipoprotein L1 gene (APOL1) with adverse kidney outcomes offers a telling example of this challenge. Certain APOL1 gene variants increase the risk of chronic kidney disease (CKD). They occur almost exclusively among people with sub-Saharan African ancestry, with highest rates in West Africa, and contribute to the higher burden of CKD among African Americans compared to European Americans. However, only a minority of people with APOL1-associated risk will develop $\mathrm{CKD}$, and no clinical measures have been shown to ameliorate the kidney disease risk associated with APOL1. Given these uncertainties, we sought to engage members of the African American public in discussion with other stakeholders about the appropriate use of APOL1 testing.

\section{Background: APOL1 Risk in African Americans}

Variants of the APOL1 gene designated as G1 and G2 are associated with increased kidney disease risk, as compared with G0, the more common form of the gene. Individuals with 2 risk variants (G1/G1, G1/G2, or G2/G2) have an estimated 2-fold greater risk of developing $\mathrm{CKD}$ and 7- to 10-fold greater risk of developing nondiabetic end-stage kidney disease (ESKD) [1-3]. Studies also indicate that transplanted kidneys from cadaveric donors with these genotypes have shorter survival $[4,5]$. Approx- imately $13 \%$ of African Americans carry 2 APOL1 risk variants and are at risk for nephropathy [6]. The risk variants occur primarily in individuals with West African ancestry, including African American, Caribbean, and some Latino populations [7]. This distribution presumably results from positive selection, because the APOL1 risk variants likely confer protection against human African trypanosomiasis (sleeping sickness) $[6,8,9]$.

Only a minority of people with $2 A P O L 1$ risk variants develops ESKD [10], indicating that other factors have an important impact on risk. However, the factors that modify APOL1-associated risk have been only partially identified. HIV infection is a potent-modifying factor [11] and is likely due to the virus eliciting production of interferon, which increases APOL1 gene expression [12]. Therapeutic administration of interferon has a similar effect. The mechanisms of kidney injury associated with $A P O L 1$ risk variants are currently being explored. Interactions between the genetic risk and comorbidities or environmental exposures remain uncertain, other than the well-documented association with HIV-AIDS and interferon therapy noted above. There are currently no measures to identify who, among those with 2 APOL1 risk variants, will develop disease and who will not.

Individuals with 2 APOL 1 risk variants who have evidence of CKD experience a more rapid loss of kidney function compared to those with 1 or zero risk variants [13-15]. In the Coronary Artery Risk Development in Young Adults study, incident albuminuria and kidney function decline were assessed over a 15-year period for European Americans, African Americans with 1 or zero risk variants ("low risk"), and African Americans with 2 risk variants "high risk" [15]. Compared to European Americans, the odds ratio for incident albuminuria was 5.71 for high risk and 2.32 for low-risk African Americans; the incidence of albuminuria for high-risk African Americans was 15.6 per 1,000 person-years [15].

Diabetes - the most common cause of ESKD in the United States - does not appear to be a modifying factor: In the Chronic Renal Insufficiency Cohort, diabetes status did not influence the rate of decline in renal function associated with $A P O L 1$ risk [14]. The role of hypertension is less certain. In the African American Study of Kidney Disease and Hypertension (AASK trial), assignment to strict versus usual blood pressure management did not change renal outcomes [14], and overall mortality rate did not differ according to APOL1 status [16]. However, in a long-term follow-up of those with 2 APOL1 high-risk variants, lower mortality was observed for people in the strict versus usual blood pressure group, indicating the 


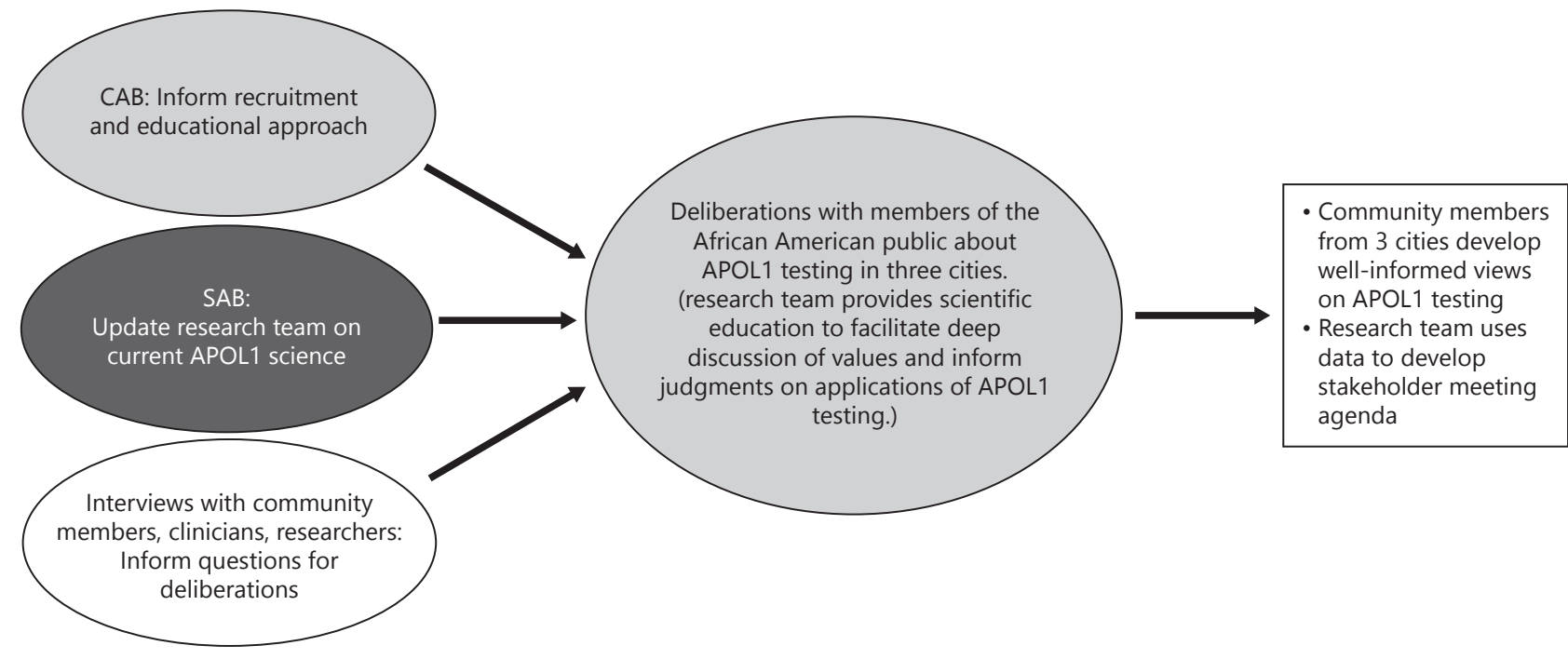

Stakeholder engagement throughout the meeting: non-hierarchical information sharing methods

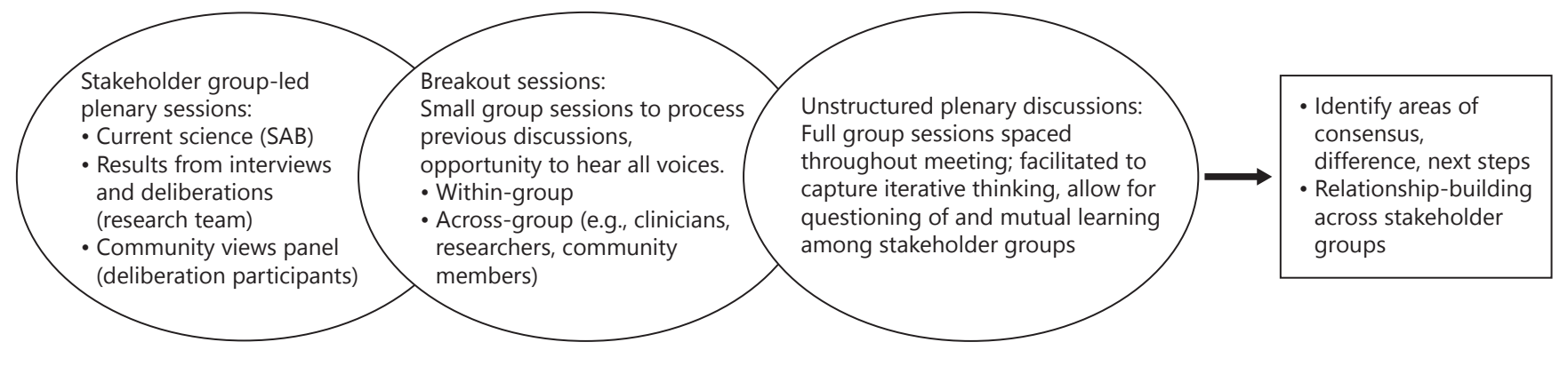

Fig. 1. Layered Engagement Process. CAB, Community Advisory Board; SAB, Scientific Advisory Board; APOL1, apolipoprotein L1.

need for further study of the benefits of intensive blood pressure management and the role of APOL1 in cardiovascular risk [16]. Furthermore, while the African American Study of Kidney Disease and Hypertension trial lasted 6 years, the effect of longer-term blood pressure control on outcome for APOL1 risk individuals remains to be determined.

Testing the APOL1 status of deceased donor kidneys prior to transplantation could optimize allocation of kidneys [17-19], directing APOL1 risk kidneys with likely shorter graft survival to those with lower life expectancy. In addition, testing of potential living donors could provide information about kidney disease risk that individuals might wish to weigh as they consider kidney donation [20]. Experts also speculate that knowledge of APOL1related risk might lead to improved control of other CKD risk factors, such as hypertension and diabetes, with po- tential patient benefit even if the actions are not specific to APOL 1 risk. Conversely, APOL1 testing in the absence of clinical benefit for that testing could lead to stigma or discrimination and could use limited health care resources that might be better utilized elsewhere. Our project sought community input on these and other potential trade-offs.

\section{Methods}

\section{Preparing for Stakeholder Discussion}

In preparation for a national meeting of community members, researchers, and other stakeholders, we took several steps to ensure that the cross-stakeholder discussion was well informed (Fig. 1). Study investigators received advice from 3 Community Advisory Boards (CABs) at study sites in Seattle WA, Nashville TN, and Jackson MS, and from a national Scientific Advisory Board comprised 
Table 1. Participants in layered engagement

a Formative interview and deliberation participants

\begin{tabular}{|c|c|c|c|c|c|c|c|}
\hline & \multicolumn{2}{|l|}{ Seattle } & \multicolumn{2}{|l|}{ Nashville } & \multicolumn{2}{|l|}{ Jackson } & $\frac{\text { National }}{\text { interviewee }}$ \\
\hline Family members* & 3 & 2 & 5 & 0 & 5 & 2 & \\
\hline Community members* & 2 & 9 & 5 & 9 & 5 & 5 & \\
\hline Nephrologists & 5 & - & 5 & - & 4 & - & \\
\hline Clinician total & 8 & - & 10 & - & 9 & - & \\
\hline Researchers $^{\varphi}$ & & & & & & & 10 \\
\hline Interview totals by site & 28 & & 25 & & 23 & & 10 \\
\hline
\end{tabular}

b National meeting participant affiliations

$\begin{array}{lc}\text { Study team } & 10 \\ \text { Community deliberants } & 8 \\ \text { CABs } & 5 \\ \text { SAB } & 7^{\#} \\ \text { Healthcare providers, researchers, advocates } & 10 \\ \text { NIH } & 7^{\#}\end{array}$

Total participants

46

\# One scientific advisory board member is also affiliated with NIH.

* All community participants self-identified as Black or African American. A patient was defined as individual with history of kidney disease; a family member was defined as relative of an individual with kidney disease; a community member was defined as self-identified Black or African American without personal or family history of kidney disease.

$\varphi$ Researchers were recruited nationally based on expertise. All were conducted by a Seattle-based interviewer.

CAB, Community Advisory Board; SAB, Scientific Advisory Board; NIH, National Institutes of Health.

of experts in APOL1-related science, nephrology, and genetic testing. Using common qualitative research practices [21], we also conducted formative interviews with 76 primary care providers, nephrologists, researchers, and African American patients and community members (Table 1), to identify key questions and knowledge needs related to $A P O L 1$ testing. These participants were recruited purposively and interviewed for 30-60 min by phone or in person about several topics regarding APOL1 testing in clinical, research, and transplantation settings. Interviews were audio recorded and transcribed. We used the initial steps in qualitative analysis (i.e., 3 teams of 2 closely read each transcript for each stakeholder group, and discussed the key content, followed by a group meeting of the 6 investigators) to develop preliminary themes to inform community deliberations and the national meeting (Table 2). Detailed coding of interview transcripts, with incorporation of data from the community deliberations and national meeting, is being conducted and will be reported separately. These steps informed planning and preparation of educational materials for 3 community deliberations [22] (one at each of the above sites) involving 39 African
Americans. We have described our deliberative methods and results elsewhere [23]. These 2-day deliberations involved diverse members of the African American community (Table 1) who were provided with a scientific introduction to APOL1 and kidney disease before considering the implications of APOL1 testing in routine patient care and kidney transplant settings. They afforded participants time to gain a deeper understanding of this novel topic, to reflect on and debate key questions related to testing in routine clinical care and kidney transplant programs with other community members, and to come to informed value judgments on several policy questions. The outcomes of these 3 deliberations included both the data we have reported elsewhere [23] and a highly informed and engaged cadre of community members, prepared to participate in cross-stakeholder conversations.

\section{Convening Stakeholders}

A stakeholder meeting was convened in March 2018 in Bethesda MD, with the meeting structure and discussion based on information derived from the interviews and deliberations 
Table 2. Main messages from formative interviews

\begin{tabular}{ll}
\hline Topic & Findings \\
\hline Information needs & $\begin{array}{l}\text { Community members, researchers, and clinicians all supported } \\
\text { the need for reliable information sources on } A P O L 1 \text { for } \\
\text { community members and clinicians }\end{array}$ \\
\hline Offer of APOL1 results obtained in research to study participants & $\begin{array}{l}\text { Community members, researchers, and clinicians all supported } \\
\text { the offer of } A P O L 1 \text { results to research participants }\end{array}$ \\
\hline Use of APOL1 testing in routine clinical care & $\begin{array}{l}\text { Clinicians and researchers were generally negative and } \\
\text { community members generally positive about the use of } A P O L 1 \\
\text { testing in routine clinical care }\end{array}$ \\
\hline Use of $A P O L 1$ testing in kidney transplant programs & $\begin{array}{l}\text { Mixed views about the use of } A P O L 1 \text { testing in kidney transplant } \\
\text { programs were expressed in all groups }\end{array}$ \\
\hline
\end{tabular}

APOL1, apolipoprotein L1.

(Fig. 1). Eight community deliberants, $5 \mathrm{CAB}$ members and 7 Scientific Advisory Board members attended the meeting. The remaining 26 participants were invited to ensure representation from diverse stakeholder groups and included genomic researchers, clinicians, health service researchers, bioethicists, patient advocates, and representatives of professional organizations and federal funding agencies (Table 1). The meeting was structured to promote interactive discussion across perspectives in both plenary and small group settings. Participants were tasked with identifying areas of consensus and disagreement about APOL1 testing and with considering needed research and actions regarding APOL1 testing in the African American community.

The 2-day meeting provided all stakeholders with a common fund of information; allowed for cross-stakeholder conversation and questioning, leading to deeper understanding of each other's perspectives; and facilitated identification of areas of agreement and disagreement. The meeting began with a scientific update and a report of findings from formative interviews and community deliberations that preceded the meeting, followed by a panel of community members sharing their perspectives. Iterative plenary and small group sessions were then used to clarify issues concerning the use of $A P O L 1$ testing in transplant programs and routine clinical care and to consider the return of $A P O L 1$ results to research participants. The first small group sessions were each composed of members from 1 to 4 stakeholder groups (community members, researchers and funders, clinicians, and advocates). This session allowed participants to confer with others who shared their experience and perspective. The second small group session assigned individuals to mixed stakeholder groups (typically composed of 1-2 members from each of the 4 stakeholder categories) to create an opportunity to share differing perspectives.

Detailed note-taking throughout the meeting allowed the research team to review results of discussions at the end of Day 1 and at the end of the morning session of Day 2, in order to generate a draft list of salient conclusions and recommendations for group discussion in plenary sessions on the afternoon of Day 2. For each

APOL1 Testing: Involving Community in Policy Discussions draft conclusion and recommendation, the team catalogued the reasons articulated in support and opposition. The meeting concluded with 2 plenary sessions in which these draft conclusions and recommendations were reviewed and discussed, with opportunities for clarifications, corrections, and further discussion. This process allowed the group to collectively generate final recommendations (Table 3). After the meeting, a written document was prepared, summarizing the conclusions and recommendations, and sent to all meeting participants for review and approval.

We name this process of meeting preparation and stakeholder discussion "layered engagement" (Fig. 1). Community member attendees came to the meeting with substantial background knowledge accrued through their participation in either a CAB or a community-based deliberation. The March 2018 meeting began with presentations intended to ensure that all participants had a shared understanding of APOL1-associated risk and the outcomes of the community deliberations.

\section{Results}

Each component of the layered engagement process added to the outcome. Formative interviews with community members, researchers, and clinicians allowed us to gauge the similarities and differences in perspectives of different stakeholders and informed the development of educational materials for community deliberations. The deliberations enabled a group of community members to become informed about APOL1 research and the potential for $A P O L 1$ testing and to debate the pros and cons of different approaches to APOL1 testing. The national meeting provided an opportunity for discussion and consensus development across different stakeholder perspectives. 
Table 3. Recommendations

\begin{tabular}{lll}
\hline Key recommendations & Details of recommendations & Discordant views \\
\hline 1. African Americans and others with & - Information about APOL1 should be & None \\
West African heritage should be & integrated with general information \\
informed about APOL1 risk & about kidney health \\
& - Partnerships among local community \\
& organizations, advocacy groups, \\
& professional organizations, academia, \\
& and public agencies offer the best \\
& opportunity to accomplish this goal \\
& - Healthcare providers who see patients \\
& with West African heritage need \\
& educational materials to assist them in \\
& addressing patient questions and \\
& requests for $A P O L 1$ testing
\end{tabular}

2. Participants in studies to evaluate $A P O L 1$ risk should be offered the opportunity to learn their APOL1 status, with appropriate counseling

3. Involvement of African American community members in development of policies and educational materials about $A P O L 1$ risk and APOL1 testing is needed to ensure that community needs and preferences are addressed

4. APOL1 testing should be integrated into kidney transplant programs
- Research is needed to ensure better understanding of APOL1 risk and necessarily involves determining participant $A P O L 1$ status

- Ongoing collaboration among community stakeholders, researchers, and clinicians is needed to guide appropriate response to emergent information about $A P O L 1$ risk
None

None

- Kidneys from deceased donors who are African American or of West African ancestry should be tested, so that APOL1 information can be incorporated into kidney allocation decisions

- Living donors who are African American should be offered testing for APOL1 risk, so that they can factor their risk status into their decision whether or not to donate a kidney
- Stakeholders had differing views about whether a transplant program should notify family members of a deceased donor if APOL1 risk is found; and about whether transplant recipients should be told the APOL1 status of deceased donor kidneys

- Stakeholders had differing views about whether transplant programs should require testing of living donors; whether a living donor who is $A P O L 1$ risk positive should be allowed to donate a kidney; and whether this information should be transmitted without explicit permission of the living donor

- Many but not all stakeholders believed that testing should be available for those who request it, on the grounds that $A P O L 1$ risk status could be taken into account as patient and doctor discuss preventive care to maintain kidney health improve outcomes for people with APOL1. However, more consideration is needed regarding clinical testing upon request

- There is a need for outreach to and educational materials for at-risk communities, primary care clinicians, and nephrologists, to provide them with information about $A P O L 1$ risk

- Concerns include the cost of APOL1 testing, who would pay for the test, and privacy, including who or what entities would be eligible to view the results

APOL1, apolipoprotein L1. 


\section{Recommendations}

Meeting conversations produced several areas of broad agreement with respect to the use of $A P O L 1$ testing in 3 areas: Kidney transplant programs, the offer to return $A P O L 1$ test results obtained in research to research participants, and community education (Table 3 ). Meeting participants agreed that APOL1 testing should be integrated into kidney transplant programs, where its clinical value in informing living donor and transplant decisionmaking is more evident but should not be routinely offered to other patients in clinical care settings at this time, where its utility remains uncertain. Participants agreed that individuals participating in APOL1-related research should be offered the opportunity to learn their status, with appropriate counseling and education, and that, as studies are completed, research updates should be communicated in plain language to African American communities and other communities with West African heritage.

This last view relates to what was perhaps the most resounding area of agreement, namely, that the African American public should be informed about the scientific discovery of APOL1. Any broad-based educational initiative would need to ensure that the information is conveyed accurately, responsibly, and effectively, so that the message reaches diverse segments of the African American public in appropriate venues and platforms. For that to happen, meeting participants urged that communities be meaningfully involved in the development of communication approaches and dissemination of materials. With its ancestry-specific origins and protective effects against trypanosomiasis, community representatives also noted that APOL1 could offer a compelling story to jumpstart a larger conversation about kidney disparities and kidney health.

Areas of disagreement also emerged, particularly with regard to how APOL1 testing is conducted in kidney transplant programs and the general availability of $A P O L 1$ testing. In the transplant context, meeting participants had differing views about sharing the APOL1 status of deceased kidney donors with donor family members and with transplant recipients. There was also lack of consensus about whether transplant programs should require living donors to be tested (as opposed to offering voluntary testing), an outcome foreshadowed in our community deliberations, and prohibit those with APOL1 risk genotypes from becoming donors. In addition, participants held differing views about whether testing should be made available to patients who request it. There was skepticism related to its unclear utility in directing treat-

APOL1 Testing: Involving Community in

Policy Discussions ment and also concerns about costs, insurance, privacy and confidentiality, and potential discrimination. Balanced against these concerns was the view held by many participants that individuals who wish to have this healthrelated information should be able to obtain it. Several community members argued that there was personal value to knowing about a health risk, even if there were not specific medical actions to be taken. At the same time, participants noted that any APOL1 testing should be accompanied by educational materials not only for the patient receiving information but also for primary care clinicians and nephrologists, to prepare them to discuss APOL1 testing with patients. This point led to acknowledgment of concerns about the cost of APOL1 testing, who would pay for the test, and the ways the information generated would be confidentially managed and specifically, who or what entities would be eligible to view the results. These concerns were identified as important issues for further research and policy development.

\section{Lessons}

Perhaps the most important lesson of the meeting related to the need for stakeholders representing different perspectives learning and talking together about APOL1 research, clinical and transplant practices, and kidney disease and disparities. All participants played an important role in the meeting; in particular, the meeting was designed to allow community participants a significant voice in the discussion and to interact directly with clini$\mathrm{cal}$ and research professionals with expertise in APOL1. The conversation did not stop at questions about whether and how APOL1 testing should be done. It extended into far more sensitive topics such as mistrust of the research and medical establishments and how to talk about race and ancestry as they relate to disease and disparities. Stories of unanticipated deaths and personal fears were shared, and significant rapport was established among participants. We believe the design of the meeting contributed to new learning for everyone in attendance. No doubt the good will and intentions of our meeting participants should get the bulk of the credit.

\section{Conclusion}

While time intensive, we believe the preparation for the meeting (Fig. 1) led to a deeper and more meaningful engagement among all meeting participants and promoted equity in the meeting room. Dedication of this time signaled respect to all involved; gave community mem- 
bers who do not have a scientific background an opportunity to develop and share well-informed, nuanced opinions; and afforded the scientific community attendees an opportunity to hear directly from those affected by their work. This layered engagement resulted in cooperatively developed recommendations for action, based on mutual understanding of broad stakeholder interests.

Medicine is faced with an increasing array of genetic information, often involving informational complexity and ambiguity regarding its clinical or personal value. If there are no specific clinical actions to be taken on the basis of a genetic test - as is the case at present when APOL1 testing is done outside the transplant context clinicians and clinical researchers may understandably conclude that testing should not be done. Our stakeholder discussion suggests that this conclusion may be too restrictive. Community members strongly supported access to APOL1 testing for those who want it. And, yet, creating opportunities for individuals to access APOL1 information must also address difficult resource questions about who pays for testing and counseling and how appropriate education and counseling are defined and responsibly and reliably provided. As research on APOL1 and kidney disease continues, new questions are likely to arise, potentially involving other challenging trade-offs. We believe the best answers to these questions will come from continued discussion among stakeholders, with concerted efforts to ensure active and informed community participation. Our meeting led to concrete suggestions for action and identified topics requiring further research and deliberation (Table 3), including a priority on developing materials to inform both community members and clinicians about APOL1-associated kidney disease risk.

However, the approach of layered engagement poses its own resource questions. Our work formed part of a funded research project. Efforts to sustain dialogue will require additional resources, as will the application of this approach to other difficult questions in clinical care. Two undertakings are required (1) methods to enable community members to be informed participants in cross-stakeholder discussions about policy options and (2) opportunities to convene stakeholders for discussion across differing perspectives and experience. Partnerships among professional and advocacy organizations, health systems, and governmental agencies may provide opportunities for cost-effective approaches to accomplish these goals. Our experience suggests that a commitment to iterative discussion over time is essential to assuring thoughtful input.

\section{Acknowledgments}

The authors acknowledge the essential contributions of each of the APOL1 Stakeholders Project Meeting participants, without whom, this work would not have been possible. Additionally, we acknowledge the interview and deliberation participants, and the following individuals who contributed to early data collection: Delia Owens, Sonya Bubar, Dr. Clarence Spigner, Anquinette Barry, and Deborah Davies.

\section{Statement of Ethics}

Institutional Review Boards at the University of Mississippi, the University of Washington, and Vanderbilt University approved the research phases of the larger study, and all interview and deliberation participants provided informed consent. Ethical approval was not required for the meeting, as it does not meet the definition of research.

\section{Disclosure Statement}

Wake Forest University Health Sciences and B.I.F.: have rights to an issued US patent related to APOL1 genetic testing. Dr. Barry I. Freedman has grant support from the NIH and is a consultant for AstraZeneca Pharmaceuticals and Renalytix AI. All other authors have no conflicts of interest to declare.

\section{Funding Sources}

This work was supported by funding from National Institutes of Health, including grant No. R01HG007879 from the National Human Genome Research Institute, grant No. Z01DK043308 from the Intramural Research Program, National Institute of Diabetes and Digestive and Kidney Diseases (NIDDK), and CTSA award No. UL1 TR002243 from the National Center for Advancing Translational Sciences. Dr. Bessie A. Young is also supported in part by funding from the Veterans Affairs Puget Sound Health Care System. Dr. Kerri L. Cavanaugh also receives grant support from the Department of Defense. Dr. Ebele M. Umeukeje is also supported by NIH grant No. 1K23DK114566-01A1 from the NIDDK. Opinions expressed in this manuscript are those of the authors and do not necessarily reflect the official views of the National Institutes of Health or the authors' institutions.

\section{Author Contributions}

B.A.Y., W.B., K.L.C., and J.G.W.: full study design and site principle investigators; E.B., E.M.U., S.M.F., and K.M.W.: interview and deliberation data collection and analysis; B.I.F. and J.B.K.: scientific content to shape data collection activities and meeting discussions; B.A.Y., W.B., E.B., S.M.F., and E.M.U.: meeting planning and facilitation; B.A.Y., W.B., E.B., S.M.F., and K.M.W.: article, table, and figure drafting. All authors participated in the critical revision of earlier drafts and recommendations and approved the final version of the manuscript. 


\section{Appendix}

Participants in the APOL1 Stakeholders Project Meeting

Larry Agodoa, MD, Office of Minority Health Research Coordination, NIDDK; Vence Bonham, JD, Social and Behavioral Research Branch, NHGRI; Shawneequa Callier, MA, JD, George Washington School of Medicine and Health Sciences; Kirk Campbell, MD, American Society of Nephrology, Icahn School of Medicine, Mount Sinai; Michael Choi, MD, National Kidney Foundation, Johns Hopkins School of Medicine, Purnell Davis, Seattle, WA; Ahmed Elmi, MPH, CHES, Division of Engagement, NIH, Moïse Findley, MPH, Nashville, TN; David Flannery, MD, American College of Medical Genetics and Genomics; Faith Fletcher, $\mathrm{PhD}$, University of Alabama School of Public Health; Raquel Greer, MD, MHS, FACP, Johns Hopkins University School of Medicine; Gentzon Hall, MD, PhD, Duke University School of Medicine; Willie Halliburton, BS, MPH, Nashville, TN; Maxine Hayes, MD, MPH, Washington State Dept of Health, retired; Ashley Hedeen, MD, VA Puget Sound Health Care System; Jonathan Himmelfarb, MD, University of Washington School of Medicine
Kidney Research Institute; Carol Horowitz, MD, MPH, Mount Sinai School of Medicine; Wendelyn Inman, $\mathrm{PhD}$, Nashville, TN; Tiffany Jones-Smith, Texas Kidney Foundation; Richard Knight, American Association of Kidney Patients; Barbara Koenig, PhD, RN, University of California San Francisco School of Nursing; Kevin McKenzie, BS, MPH, Nashville, TN; Martin Pollak, MD, Harvard University Medical School, Beth Israel Medical Center; Kenneth Poston, BS, Seattle, WA; William Powell, MTS, Nashville, TN; Susan Quaggin, MD, American Society of Nephrology, Northwestern University School of Medicine; Nishadi Rajapakse, PHD, MHS, National Institute on Minority Health and Health Disparities, NIH; Glenda Roberts, Kidney Research Institute, University of Washington; Mimsie Robinson, MA, MPS, Bethel Gospel Assembly, Living Water Christian Center; Charles Rotimi, PhD, Metabolic, Cardiovascular and Inflammatory Disease Genomics Branch, Center for Research on Genomics and Global Health, NIH, Nia Sims, Mississippi State University; Nannette Ward, AA, Seattle, WA; Cheryl Winkler, PhD, Frederick National Laboratory, Basic Research Laboratory, Center for Cancer Research, National Cancer Institute, NIH.

\section{References}

1 Anders HJ, Rovin B. A pathophysiologybased approach to the diagnosis and treatment of lupus nephritis. Kidney Int. 2016 Sep; 90(3):493-501.

2 Foster MC, Coresh J, Fornage M, Astor BC, Grams M, Franceschini N, et al. APOL1 variants associate with increased risk of $\mathrm{CKD}$ among African Americans. J Am Soc Nephrol. 2013 Sep;24(9):1484-91.

3 Pollak MR, Genovese G, Friedman DJ. APOL1 and kidney disease. Curr Opin Nephrol Hypertens. 2012 Mar;21(2):179-82.

4 Freedman BI, Pastan SO, Israni AK, Schladt D, Julian BA, Gautreaux MD, et al. APOL1 Genotype and Kidney Transplantation Outcomes From Deceased African American Donors. Transplantation. 2016 Jan;100(1):194202.

5 Freedman BI, Limou S, Ma L, Kopp JB. APOL1-Associated Nephropathy: A Key Contributor to Racial Disparities in CKD. Am J Kidney Dis. 2018 Nov;72(5S1):S8-16.

6 Wasser WG, Tzur S, Wolday D, Adu D, Baumstein D, Rosset S, et al. Population genetics of chronic kidney disease: the evolving story of APOL1. J Nephrol. 2012 Sep-Oct; 25(5):603-18.

7 Nadkarni GN, Gignoux CR, Sorokin EP, Daya M, Rahman R, Barnes KC, et al. Worldwide Frequencies of APOL1 Renal Risk Variants. N Engl J Med. 2018 Dec;379(26):2571-2.

8 Genovese G, Friedman DJ, Ross MD, Lecordier L, Uzureau P, Freedman BI, et al. Association of trypanolytic ApoL1 variants with kidney disease in African Americans. Science. 2010 Aug;329(5993):841-5.
9 Riella C, Siemens TA, Wang M, Campos RP, Moraes TP, Riella LV, et al. APOL1-Associated Kidney Disease in Brazil. Kidney Int Rep. 2019 Mar;4(7):923-9.

10 Mukamal KJ, Tremaglio J, Friedman DJ, Ix JH, Kuller LH, Tracy RP, et al. APOL1 Genotype, Kidney and Cardiovascular Disease, and Death in Older Adults. Arterioscler Thromb Vasc Biol. 2016 Feb;36(2):398-403.

11 Kasembeli AN, Duarte R, Ramsay M, Mosiane P, Dickens C, Dix-Peek T, et al. APOL1 Risk Variants Are Strongly Associated with HIV-Associated Nephropathy in Black South Africans. J Am Soc Nephrol. 2015 Nov;26(11): 2882-90.

12 Nichols B, Jog P, Lee JH, Blackler D, Wilmot M, D'Agati V, et al. Innate immunity pathways regulate the nephropathy gene Apolipoprotein L1. Kidney Int. 2015 Feb;87(2):332-42.

13 Chen TK, Tin A, Peralta CA, Appel LJ, Choi MJ, Lipkowitz MS, et al. APOL1 Risk Variants, Incident Proteinuria, and Subsequent eGFR Decline in Blacks with HypertensionAttributed CKD. Clin J Am Soc Nephrol. 2017 Nov;12(11):1771-7.

14 Parsa A, Kao WH, Xie D, Astor BC, Li M, Hsu CY, et al.; AASK Study Investigators; CRIC Study Investigators. APOL1 risk variants, race, and progression of chronic kidney disease. N Engl J Med. 2013 Dec;369(23):218396.

15 Peralta CA, Bibbins-Domingo K, Vittinghoff E, Lin F, Fornage M, Kopp JB, et al. APOL1 Genotype and Race Differences in Incident Albuminuria and Renal Function Decline. J Am Soc Nephrol. 2016 Mar;27(3):887-93.
$16 \mathrm{Ku} \mathrm{E}$, Lipkowitz MS, Appel LJ, Parsa A, Gassman J, Glidden DV, et al. Strict blood pressure control associates with decreased mortality risk by APOL1 genotype. Kidney Int. 2017 Feb;91(2):443-50.

17 Julian BA, Gaston RS, Brown WM, ReevesDaniel AM, Israni AK, Schladt DP, et al. Effect of Replacing Race With Apolipoprotein L1 Genotype in Calculation of Kidney Donor Risk Index. Am J Transplant. 2017 Jun;17(6): $1540-8$.

18 Riella LV, Sheridan AM. Testing for HighRisk APOL1 Alleles in Potential Living Kidney Donors. Am J Kidney Dis. 2015 Sep;66(3): 396-401.

19 Freedman BI, Julian BA. Should kidney donors be genotyped for APOL1 risk alleles? Kidney Int. 2015 Apr;87(4):671-3.

20 Doshi MD, Ortigosa-Goggins M, Garg AX, Li L, Poggio ED, Winkler CA, et al. APOL1 Genotype and Renal Function of Black Living Donors. J Am Soc Nephrol. 2018 Apr;29(4): 1309-16.

21 Patton MQ, Patton MQ. Qualitative research and evaluation methods. 3rd edition. Thousand Oaks, CA: Sage Publications; 2002. p. xxiv, 598, 65 .

22 Blacksher E, Diebel A, Forest PG, Goold SD, Abelson J. What is public deliberation? Hastings Cent Rep. 2012 Mar-Apr;42(2):14-7.

23 Umeukeje EM, Young BA, Fullerton SM, Cavanaugh $\mathrm{K}$, Owens D, Wilson JG, et al. You Are Just Now Telling Us About This? African American Perspectives of Testing for Genetic Susceptibility to Kidney Disease. J Am Soc Nephrol. 2019 Apr;30(4):526-30. 